\title{
Communication-based Leashing of Real Flying Robots
}

\author{
Sabine Hauert*, Severin Leven*, Jean-Christophe Zufferey, Dario Floreano
}

\begin{abstract}
Aerial robots are often required to remain within the communication range of a base station on the ground to exchange commands, sensor data or as a safety mechanism. For this purpose, we propose a minimal control strategy for steering flying robots using only communication hardware (e.g. WiFi module or radio modem) instead of GPS or cameras. To avoid being dependent on the specifics of the communication hardware or its driver, we propose to measure the number of messages the robot receives from the base as a control input. Leashing is then performed by having the robot react to low message rates by moving towards the base in order to improve the communication. Results show both in theory and reality that this strategy can leash the robot to the base in scenarios with limited wind or base mobility.
\end{abstract}

\section{INTRODUCTION}

Ensuring wireless communication between a flying robot and a base station on the ground is an intrinsic requirement to receive commands, transmit sensor data or relay information [1]-[5]. In this paper, we present a strategy to leash a physical flying robot to a base so that it remains within its communication range. Leashing can also be used as a safety mechanism to ensure that a robot does not get lost by flying too far away from the base. This is especially interesting for testing new research algorithms on a robot or for learning how to pilot.

Previous research aimed at maintaining a flying robot within communication range of a base by setting a predetermined limit to the distance from the base at which the robot can fly [1], [6]. However, distance has been shown to correlate poorly with the quality of successful radio transmissions because the propagation of radio signals depends on the environment (weather, reflections, interferences) [7]. Instead, using the radio module itself for controlling the behavior of the robot (communication-based behavior) is appealing since it directly relates to the capacity of the robot to send and receive radio messages. Moreover, the robot does not require additional sensors for navigation. As a result, it is freed from positioning information typically derived from GPS and may therefore work in situations where the GPS signal is not available (occluded or extra-terrestrial environments). This is novel given that the use of position information for flying robots is standard practice in current research [8]-[12].

Communication-based behaviors for flying robots have been investigated by Dixon et al. [4] who used the signal-tonoise ratio (SNR) of incoming radio signals to determine if

\footnotetext{
Laboratory of Intelligent Systems, Ecole Polytechnique Fédérale de Lausanne, Switzerland \{sabine.hauert, severin.leven, jean-christophe.zufferey, dario.floreano\} @epfl.ch

*These authors contributed equally to this work.
}

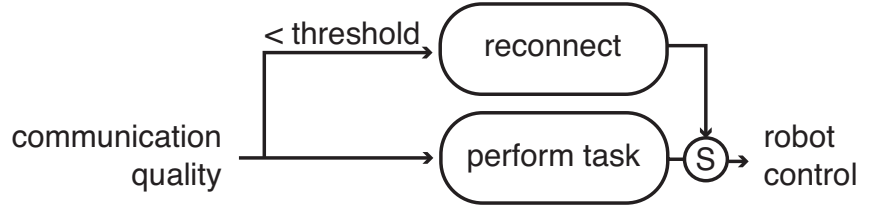

Fig. 1. Communication-based leashing controller for a robot using the subsumption architecture [13]. Here, the reconnection behavior takes priority over the robot's task when the communication quality is too low.

a robot is in an optimal location to communicate with a base or other robots. However, experiments on real flying robots relied on position information to function [5]. Furthermore, SNR indications are not always made available by the radio module. Indeed, SNR information is treated at the driver level and whether or not this information is passed up to the user depends on which off-the-shelf radio module is used. In addition, even if the SNR is given, the source of the signal might not be specified. This is problematic when multiple emitters are present.

In this paper, we propose a simple communication-based behavior that exclusively relies on the rate of messages successfully received by a robot from a base. For this purpose, the base broadcasts small "hello messages" at a regular interval. Measuring the rate of incoming messages is always feasible, regardless of the radio module used. The robot is leashed to the base by allowing it to move freely as long as the message rate is high, and pulling the robot back towards the base when the extent of the leash has been reached (low message rate). The manner in which the leashing is performed highly depends on the dynamics of the platform and the noise present in the environment. Real-life conditions are such that there are often disturbing relative displacements between the base and robot due to wind or base mobility. Therefore, we theoretically identify regimes where the proposed approach can function. We then show, within these regimes, the leashing of a real fully autonomous fixed-wing platform.

\section{ROBOT CONTROL}

Communication-based leashing looks at how to maintain a robot within the communication range of a radio emitter using the communication hardware as the only sensor. The robot navigates freely according to its assigned task (e.g. exploration, tracking) as long as the quality of the communication between the base and robot is good, and performs a reconnection behavior when it degrades below a certain threshold (Fig. 1).

In this paper, we control the turn rate of a robot flying at 

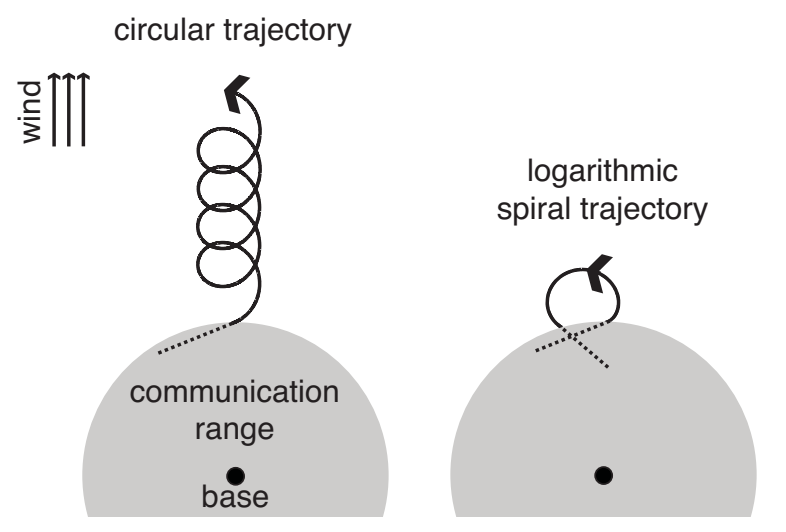

Fig. 2. The effect of wind on the trajectory of a robot performing a circular trajectory and a logarithmic spiral. Notice that the robot performing the spiral is able to reconnect to the base while the one performing a circular trajectory drifts away.

constant speed and altitude based on the rate of messages successfully received by the robot from the base (communication quality). The manner in which the reconnection is performed depends largely on the environment and on the dynamics of the robot. In Nembrini et al. [14] for example, wheeled robots advance straight until they break their communication link with neighboring robots, at which point they turn on the spot for $180^{\circ}$ and backtrack. However, unlike ground robots or rotorcrafts that can stop and turn on the spot, fixed-wing aircrafts must always remain in forward motion to stay in the air. Furthermore, aerial robots are subject to wind which displaces them with respect to the base. The most obvious reconnection behavior would be to have the robot turn-around by performing a circular trajectory with a constant turn rate. However, this leads to situations where the robot is continuously pushed away by the wind and is therefore unable to reconnect to the base (Fig. 2).

To counter this effect, we propose to use logarithmic spirals as reconnection trajectories because they can compensate for displacements between the base and robot by expanding. As an example, Fig. 3 shows how a robot following a spiral trajectory will behave with strong wind in different directions. Rather than being pushed away from the spiral starting point, the robot remains anchored. Other spirals such as archimedean curves have been studied in the literature as random search patterns for robots [15]. However, these spirals are not able to compensate a constant amount of wind in all directions and are thus unsuited as a reconnection trajectory.

We propose to theoretically determine the parameters of the spiral to be performed by the flying robot and the amount of wind that it can tolerate. The theoretical wind tolerance $v_{\text {wind }}$ is measured by the spiral expansion, which is defined as the distance between two points on the spiral which are spaced by $\Delta \theta=2 \pi$ divided by the time $t(\theta)$ needed for traveling between these points (Fig. 4). Starting from the polar equation of a logarithmic spiral with coefficients $a$ and $b$ and symbols defined in Fig. 4,

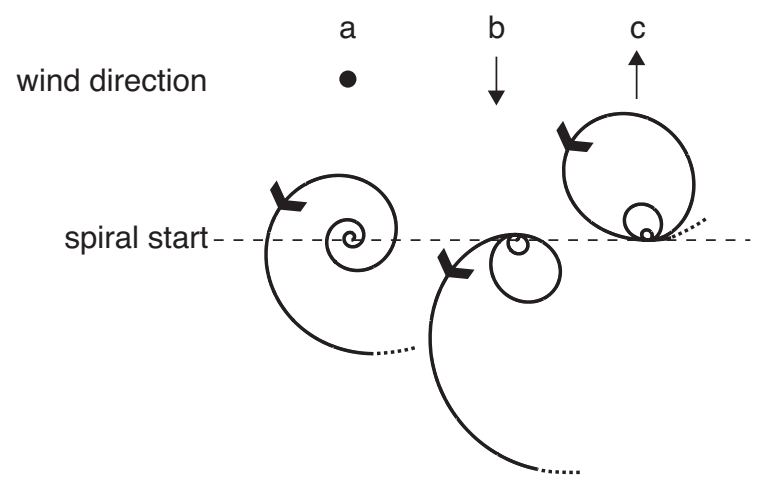

Fig. 3. The trajectory of a robot performing a logarithmic spiral when no wind is present (a) or under the influence of wind to the South (b) or North (c). Notice that the spirals remain anchored to one position rather than drift away.

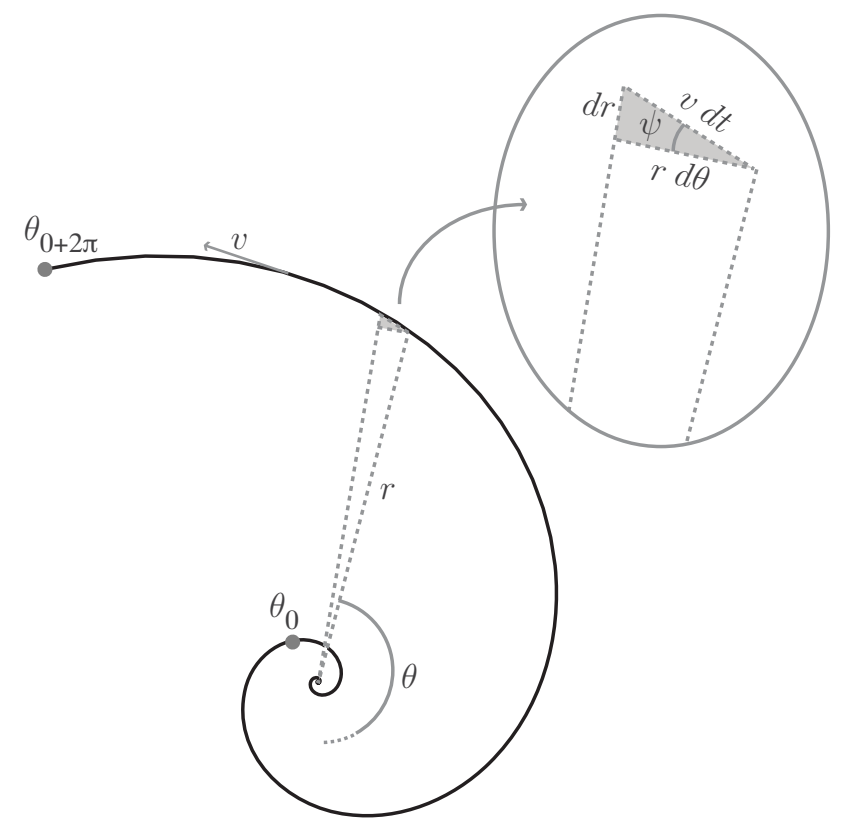

Fig. 4. Logarithmic spiral trajectory where $\theta$ is the angle and $r$ is the distance traveled from its origin. $\theta_{0}$ is the angle at which the robot starts its trajectory and $v$ is the speed of the robot.

$$
r(\theta)=a \cdot e^{b \theta},
$$

we can define the wind tolerance of the spiral as being

$$
v_{\text {wind }}=\frac{r\left(\theta_{0}+2 \pi\right)-r\left(\theta_{0}\right)}{t\left(\theta_{0}+2 \pi\right)-t\left(\theta_{0}\right)} .
$$

To solve this equation, we need to determine $t(\theta)$. Considering the local relationship of spiral variables (Fig. 4), we derive with (1)

$$
\begin{gathered}
\tan \psi=\frac{d r}{r \cdot d \theta}=b \\
r \cdot d \theta=v \cdot \cos \psi \cdot d t .
\end{gathered}
$$




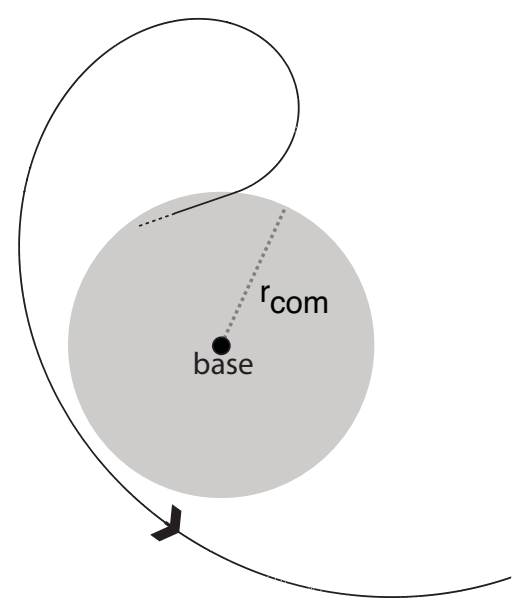

Fig. 5. The size of the spiral is constrained by the communication range of the base $r_{\mathrm{com}}$ to avoid that the reconnection behavior fails, as shown here.

For the general initial condition of a logarithmic spiral, $t=$ $0 \mathrm{~s} \Rightarrow \theta=-\infty$, we obtain by integration of (4)

$$
\frac{a}{b} \cdot e^{b \theta}=v \cdot \cos \psi \cdot t
$$

leading to

$$
t(\theta)=\frac{a}{b} \cdot \frac{e^{b \theta}}{v \cdot \cos \psi}
$$

with (3) we obtain

$$
\cos \psi=\frac{1}{\sqrt{1+\tan ^{2} \psi}}=\frac{1}{\sqrt{1+b^{2}}},
$$

and finally, based on (1), (2), (6) and (7) we find

$$
v_{\text {wind }}=\frac{b \cdot v}{\sqrt{1+b^{2}}} .
$$

Theoretically, one could parameterize the spiral with a large $b$, resulting in a tolerance to wind speeds near the speed of the robot. However, large values of $b$ lead to spirals that take too long to perform and could be too big to allow for a reconnection to the base (Fig. 5). For this reason, we impose that the distance between the initial point at which the robot starts the spiral at $\theta_{0}$ and the point at $\theta_{0}+2 \pi$ be equal to the communication range of the base station $r_{\text {com }}$. This condition ensures that the robot is not more than $2 \cdot r_{\text {com }}$ away from its disconnection point, even if wind is displacing the robot by $r_{\text {com }}$ between $t\left(\theta_{0}\right)$ and $t\left(\theta_{0}+2 \pi\right)$. Therefore, the parameter $b$ must satisfy the condition

$$
\begin{aligned}
r_{\mathrm{com}} & =r\left(\theta_{0}+2 \pi\right)-r\left(\theta_{0}\right) \\
& =a \cdot e^{b \theta_{0}} \cdot\left(e^{2 b \pi}-1\right) .
\end{aligned}
$$

To solve this equation, we first need to obtain the initial condition $\theta_{0}$, which is given by the maximum turn rate $\omega_{0}$ of the considered platform. Based on (6) we derive

$$
\theta(t)=\frac{1}{b} \cdot \ln \left(\frac{b}{a} \cdot v \cdot \cos \psi \cdot t\right)
$$

such that

$$
\frac{d \theta}{d t}=\omega=\frac{1}{b \cdot t} \Rightarrow \omega_{0}=\frac{1}{b \cdot t_{0}} .
$$

Then, using (7), (11) and (12) we obtain

$$
\theta_{0}=\frac{1}{b} \cdot \ln \left(\frac{v}{a \cdot \omega_{0} \cdot \sqrt{1+b^{2}}}\right) .
$$

This yields with (10)

$$
r_{\mathrm{com}}=\frac{v \cdot\left(e^{2 b \pi}-1\right)}{w_{0} \cdot \sqrt{1+b^{2}}},
$$

which must be solved numerically for $b$.

Given the time $t_{s}$ that elapsed since the robot started the spiral at $t_{0}$, and the maximum turn rate $w_{0}$ of the robot, we can define a turn rate controller for the robot as:

$$
\omega\left(t_{s}\right)=\frac{1}{b \cdot\left(t_{0}+t_{s}\right)}
$$

where, using (12),

$$
t_{0}=\frac{1}{\omega_{0} \cdot b} .
$$

Using these theoretical developments, each robot controller is designed for a regime that describes the conditions for which the behavior will function. In particular, the regime here is described as the minimum communication range of the base and maximum amount of wind in the environment. Note that, the faster the robot and the larger the communication range of the base, the higher the wind tolerance. While the focus here has been on wind, other sources of displacements such as a moving base can directly be addressed in the same manner.

\section{EXPERIMENTAL SETUP}

The communication-based behavior was implemented on board a fixed-wing aerial robot developed in the scope of the SMAVNET project ${ }^{1}$ and now commercialized by senseFly ${ }^{2}$. Tests were conducted outdoors in a fully autonomous manner. We hereby introduce the flying platform, the base station and the radio module used in the experiments presented in this paper.

\section{A. Flying Platform}

We use a light weight (420 g) and safe flying platform that has been developed for the rapid-prototyping of aerial robot experiments. The flying-wing configuration shown in Fig. 6 has an $80 \mathrm{~cm}$ wingspan. It is built out of Expanded Polypropylene (EPP) with an electric motor mounted at the back and two control surfaces serving as elevons (combined ailerons and elevator). The robot is equipped with an autopilot for the control of altitude, airspeed and turn rate that provides an interface for receiving commands from a navigation controller [16]. Embedded in the autopilot is a micro-controller that runs a minimalist control strategy

\footnotetext{
${ }^{1}$ The SMAVNET project aims at creating swarms of flying robots for creating communication networks in disaster areas (http:/lis.epfl.ch/smavs)

${ }^{2}$ http://www.sensefly.com/
} 


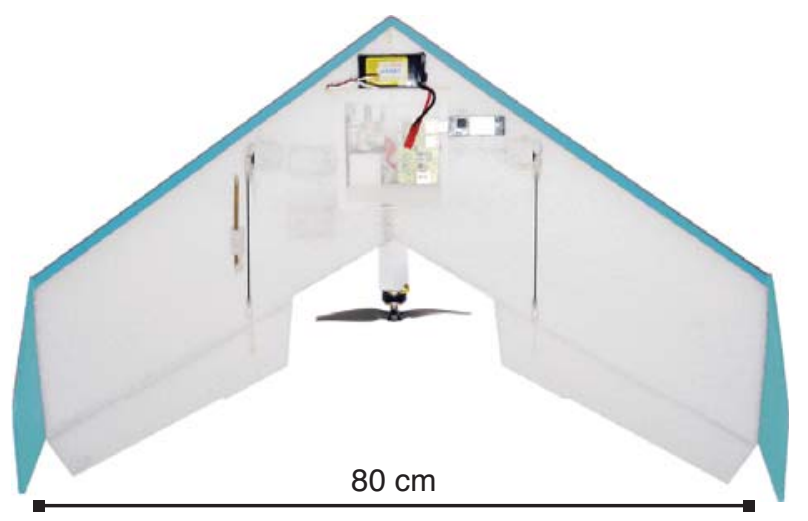

Fig. 6. Safe flying wing for outdoor experiments made out of soft material and with a back-mounted propeller. The robot is equipped with an autopilot, embedded Linux, WiFi dongle and GPS (only for logging purposes).

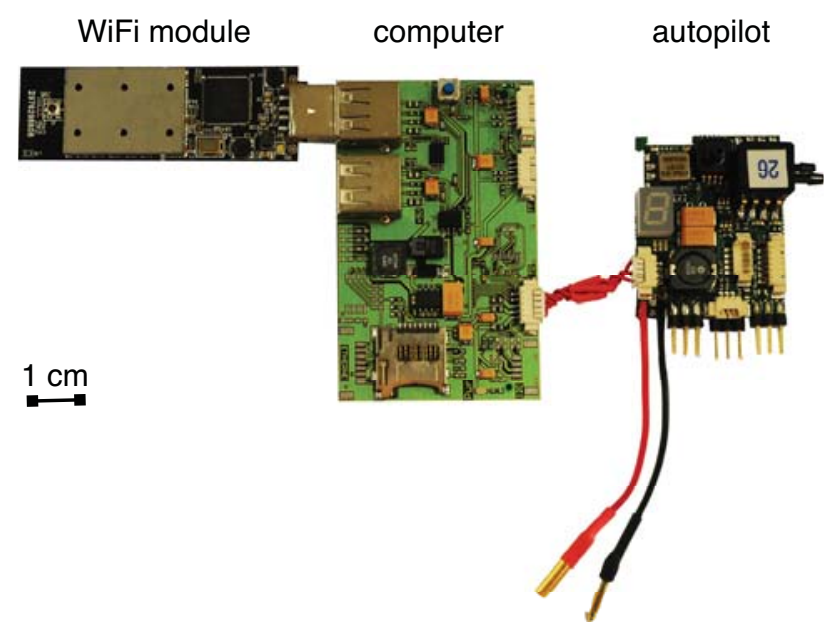

Fig. 7. Control electronics including the autopilot and the adapter-card interfacing to the Colibri Linux board and the USB WiFi dongle.

based on input from only 3 sensors: one gyroscope and two pressure sensors.

The communication-based algorithm is implemented on a Toradex Colibri PXA270 CPU board running Linux, connected to an off-the-shelf USB WiFi dongle (Fig. 7). The output of this high-level computer, namely a desired turn rate, is sent as control command to the autopilot. Altitude and airspeed commands during the experiments remained constant at $70 \mathrm{~m}$ and $12 \mathrm{~m} / \mathrm{s}$, respectively. In order to $\log$ flight trajectories, the robot is further equipped with a u-blox ${ }^{3}$ LEA-5H GPS module.

For the WiFi communication, Netgear ${ }^{4}$ WNDA3100 dongles were used that implement the 802.11n standard and transmit in the $5 \mathrm{GHz}$ band. This is interesting with respect to transmissions in the $2.4 \mathrm{GHz}$ band because it allows for less interference with the considerable number of devices currently used in this band. Dongles are configured for adhoc mode and have a communication range of nearly 500 $\mathrm{m}$ line-of-sight. For the purpose of the experiments reported

\footnotetext{
${ }^{3}$ http://www.u-blox.com

${ }^{4}$ http://netgear.com
}
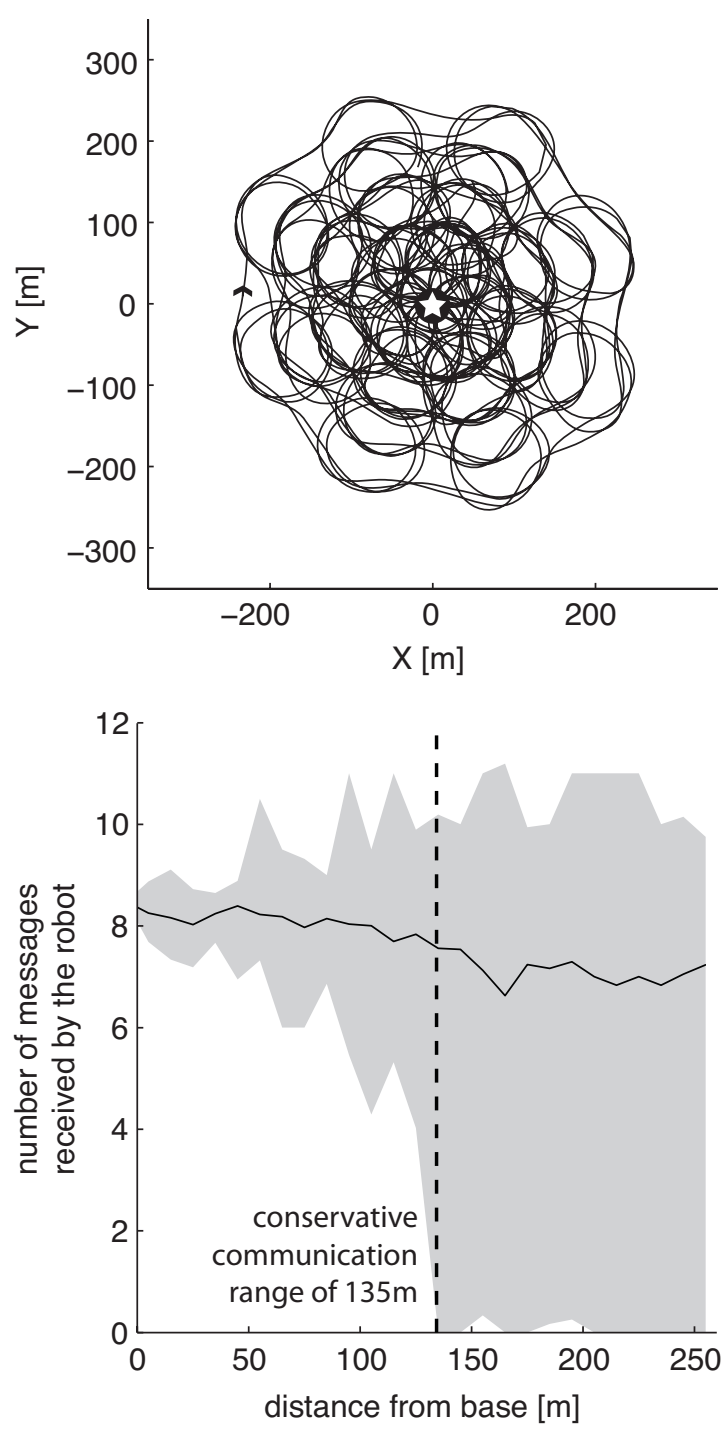

Fig. 8. Characterization of the communication between the base (star) and robot. The top graph shows the trajectory of the robot performing the characterization. The bottom graph shows the minimum, median and maximum number of messages received by the robot over $50 \mathrm{~ms}$ as a function of distance from the base.

in this paper, we modified the driver of the WiFi module to reduce the communication range of the base.

\section{B. Base Station}

The same Linux computer and WiFi dongle were used for the base station and the flying robot to ensure that the experiments presented here can easily be extended to more complex scenarios involving multiple robots. The base station is contained in a small box and can easily be transported and positioned in any environment.

\section{RESULTS}

We progressed by first identifying the minimal communication range of the base. We then used it to parameterize the reconnection behavior (by setting $b$ of the spiral in (1)) and identify the amount of wind the behavior can tolerate. Within this regime, we then demonstrated that the robot is able to 
remain leashed to the base over long periods of time and even in different locations than where the communication was characterized. This was demonstrated in scenarios with wind and a mobile base.

\section{A. Communication Characterization}

There are many different approaches to estimating the minimum communication range of the base. This can be done by making a conservative guess based on knowledge of the radio module, or by relying directly on the value given in the data-sheet. Because of changes made to our radio module driver, we chose to characterize the radio environment experimentally. This was done using a flying robot that followed the predefined pattern shown in Fig. 8 (top) so as to cover a sufficiently large area around the base. Every $50 \mathrm{~ms}$, the robot recorded the number of messages it received from the base and its position. The area was then segmented into bins of $10 \mathrm{~m} \times 10 \mathrm{~m}$ and the average number of messages received per bin over 3 separate flights was recorded. Only bins over which the robot navigated were considered for statistics. We then plot the median, minimum and maximum number of messages recorded by the bins as a function of distance. As shown in Fig. 8 (bottom), the minimum communication range for our experiments equals $135 \mathrm{~m}$ and corresponds to the first bin with a message rate of zero. This is thought to be a very conservative estimate.

Using (14), we calculated $b=0.3732$. The resulting controller can thus resist relative displacements up to a speed of $4.1957 \mathrm{~m} / \mathrm{s}(8)$. These constraints define the regime in which we use our controller for the remaining experiments.

\section{B. Communication-based Leashing}

Fig. 9 shows the trajectory of our aircraft performing autonomous communication-based leashing. During an entire 20 min flight, the robot was successful at spiraling towards the base when disconnected ${ }^{5}$. All of the 137 disconnections were successfully countered even though wind between 0.5 $\mathrm{m} / \mathrm{s}$ and $2.4 \mathrm{~m} / \mathrm{s}$ with varying direction between $220^{\circ}$ and $10^{\circ}$ was present. Interestingly, there is an unusual point in $(-10$ $\mathrm{m}, 150 \mathrm{~m}$ ) where the robot does not receive messages. This point was situated exactly above the only house within that experiment environment. Abrupt changes in the communication landscape have the same effect as a relative displacement of the robot with respect to the base and therefore need to be mitigated by the logarithmic spiral trajectories. Overall, disconnections from the base have a median value of 0.8 seconds (Fig. 10). Long disconnections of up to 26 seconds were recorded on rare occasions, followed by successful reconnections.

Moreover, it is interesting to see the large range of disconnection distances which is between $120 \mathrm{~m}$ and 258 $\mathrm{m}$, further backing the case that one can not rely on distance to predict communication quality. In addition, the median

\footnotetext{
${ }^{5}$ Robots are considered disconnected as soon as they stop receiving messages and are considered reconnected when the number of messages received during $50 \mathrm{~ms}$, low-pass filtered with a time constant of $2 \mathrm{~s}$, surpasses 5 (10 being the maximum).
}

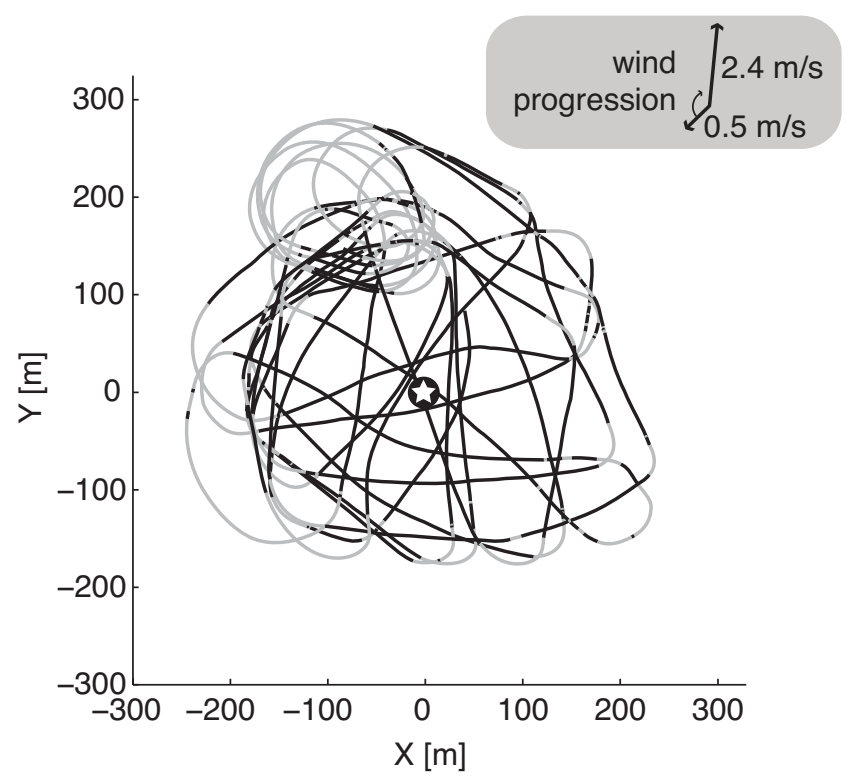

Fig. 9. 20 min trajectory of a fully autonomous flying robot leashed to a base (star) in an outdoor experiment with $0.5 \mathrm{~m} / \mathrm{s}$ to $2.4 \mathrm{~m} / \mathrm{s}$ wind between $220^{\circ}$ and $10^{\circ}$ from the North. Here the robot flies at constant speed and $70 \mathrm{~m}$ altitude. Light grey lines indicate sections of the trajectory where the robot is reconnecting using the spiraling behavior while black lines indicate that the robot is performing its assigned task, in this case, straight flight.
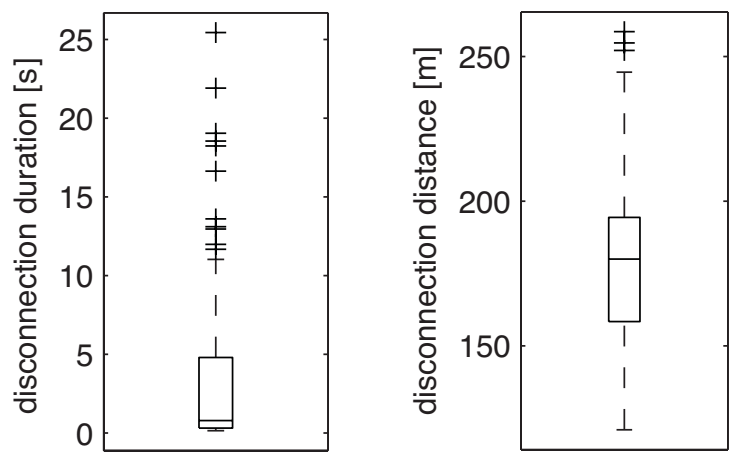

Fig. 10. Boxplot showing the duration of disconnections and the 2D distance from the base at which they occur over 137 samples for the scenario with a static base.

communication radius is around $180 \mathrm{~m}$ which is, as expected, larger than our conservative measurement of $135 \mathrm{~m}$ (Sec. IVA).

Finally, to show that communication-based leashing can directly be applied to different scenarios without any change to the controller, we present a scenario where an additional displacement is caused by a mobile base. Fig. 11 presents the trajectory of the robot when the base moves for $9 \mathrm{~min}$ along a road at a speed of $1 \mathrm{~m} / \mathrm{s}$ with front wind between 1.1 and $1.8 \mathrm{~m} / \mathrm{s}$. As before, the robot was able to mitigate disconnections and therefore follow the base.

\section{CONCLUSION}

We have demonstrated the leashing of a real flying robot to a base on the ground using only a standard radio module (WiFi) as a sensor, unlike existing research which relies on 


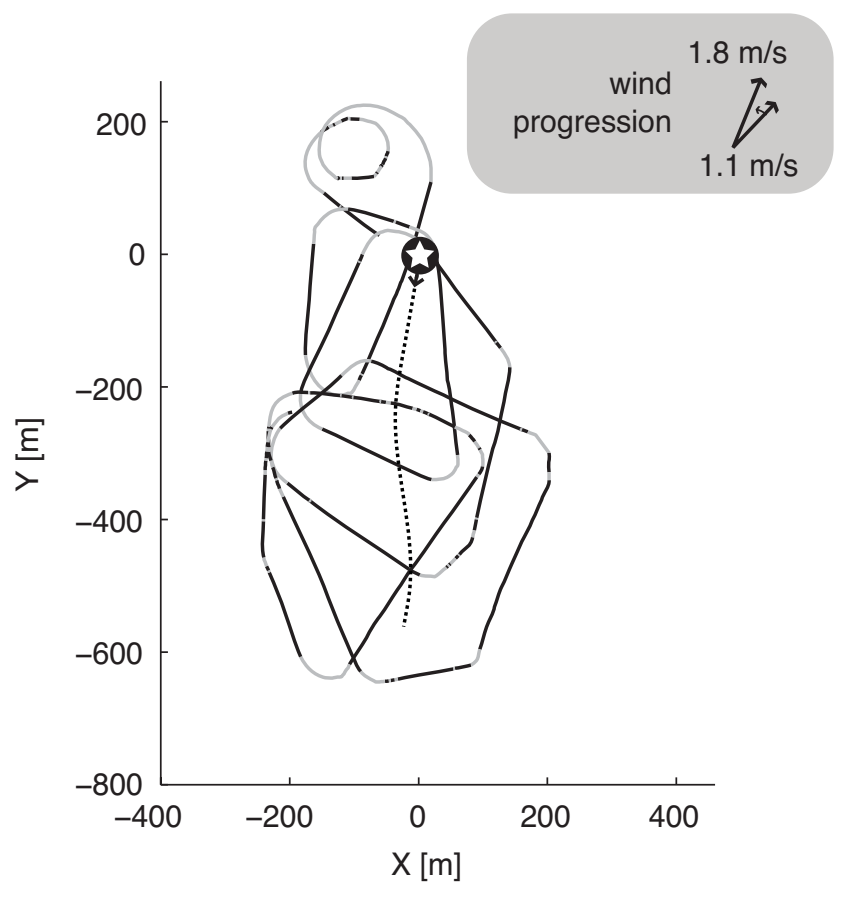

Fig. 11. 9 min trajectory of a fully autonomous flying robot leashed to a base (star) moving to the South at $1 \mathrm{~m} / \mathrm{s}$ along a road in an outdoor experiment with wind pushing the robot to the North-East at a speed between $1.1 \mathrm{~m} / \mathrm{s}$ and $1.8 \mathrm{~m} / \mathrm{s}$. Here the robot flies at constant speed and $70 \mathrm{~m}$ altitude.

GPS and explicit distance estimates. Using the proposed controller, a robot navigates freely when the rate of successfully received messages from the base is high and performs a reconnection behavior otherwise. The message rate is an easy to use metric that directly reflects the ability of a robot to receive messages and is always measurable independently of the radio module used.

In order to mitigate the effect of wind and the dynamics of the robot, we have designed a reconnection behavior based on logarithmic spiraling. This spiral has the property that it can tolerate wind by avoiding that the robot be pushed away from the base. Through a theoretical approach we are able to automatically parameterize the spiral for a given minimal communication range of the base and define the maximum wind the system can tolerate. More generally, the resulting controller can resist to other disturbing displacements between the base and robot.

In the future, we aim towards eliminating complete disconnections from the base by making the robots turn around earlier. Furthermore, we aim at extending our approach to a general investigation of reactive behaviors for flying robots based on the logarithmic spiral. In particular, we are currently deploying a swarm of Micro-Air Vehicles for Networking applications (SMAVNET project) [17], [18].

\section{ACKNOWLEDGMENTS}

This work is supported by armasuisse, competence sector Science + Technology for the Swiss Federal Department of Defense, Civil Protection and Sports.

\section{REFERENCES}

[1] P. Basu, J. Redi, and V. Shurbanov, "Coordinated flocking of UAVs for improved connectivity of mobile ground nodes," in Proceedings of the IEEE Military Communications Conference, vol. 3, 2004, pp. $1628-1634$.

[2] B. A. Kadrovach and G. B. Lamont, "Design and analysis of swarmbased sensor systems," in Proceedings of the IEEE Midwest Symposium on Circuits and Systems, vol. 1, 2001, pp. 487-490.

[3] E. Kuiper and S. Nadjm-Tehrani, "Mobility models for UAV group reconnaissance applications," in Proceedings of the IEEE International Conference on Wireless and Mobile Communications, 2006, p. 33.

[4] C. Dixon and E. W. Frew, "Maintaining optimal communication chains in robotic sensor networks using mobility control," Mobile Networks and Applications Journal, vol. 14, no. 3, pp. 281-291, 2009.

[5] E. W. Frew, C. Dixon, J. Elston, and M. Stachura, "Active sensing by unmanned aircraft systems in realistic communication environments," in IFAC Workshop on Networked Robotics, 2009.

[6] W. M. Spears, D. F. Spears, R. Heil, W. Kerr, and S. Hettiarachchi, "An overview of physicomimetics," in Simulation of Adaptive Behaviour, Workshop on Swarm Robotics, ser. Lecture Notes in Computer Science, vol. 3342. Berlin: Springer, 2005, pp. 84-97.

[7] K.-H. Kim and K. G. Shin, "On accurate measurement of link quality in multi-hop wireless mesh networks," in Proceedings of the Annual International Conference on Mobile Computing and Networking, 2006, pp. 38-49.

[8] R. W. Beard, T. W. Mclain, D. B. Nelson, D. Kingston, and D. Johanson, "Decentralized cooperative aerial surveillance using fixed-wing miniature UAVs," Proceedings of the IEEE, vol. 94, no. 7, pp. 13061324,2006

[9] D. Cole, A. Goktogan, and S. Sukkarieh, The demonstration of a cooperative control architecture for UAV teams, ser. Springer Tracts in Advanced Robotics. Berlin: Springer, 2006, vol. 39, pp. 501-510.

[10] J. Allred, A. B. Hasan, S. Panichsakul, W. Pisano, P. Gray, J. Huang, R. Han, D. Lawrence, and K. Mohseni, "SensorFlock: an airborne wireless sensor network of micro-air vehicles," in Proceedings of the 5th International Conference on Embedded Networked Sensor Systems. New York: ACM Press, 2007, pp. 117-129.

[11] J. How, E. King, and Y. Kuwata, "Flight demonstrations of cooperative control for UAV teams," in AIAA 3rd Unmanned Unlimited Technical Conference, Workshop and Exhibit, no. September, 2004, AIAA paper 2004-6490.

[12] J. Gancet, G. Hattenberger, R. Alami, and S. Lacroix, "Task planning and control for a multi-UAV system: architecture and algorithms," in Proceedings of the IEEE International Conference on Intelligent Robots and Systems, 2005, pp. 1017-1022.

[13] R. Brooks, "A robust layered control system for a mobile robot," IEEE Journal of Robotics and Automation, vol. 2, no. 1, pp. 14-23, 1986.

[14] J. Nembrini, A. Winfield, and C. Melhuish, "Minimalist coherent swarming of wireless networked autonomous mobile robots," in From Animals to Animats 7, Proceedings of the 7th International Conference on Simulation of Adaptive Behavior. Cambridge, MA: MIT Press, 2002, pp. 273-382.

[15] A. T. Hayes, A. Martinoli, and R. M. Goodman, "Swarm robotic odor localization: Off-line optimization and validation with real robots," Robotica, vol. 21, no. 4, pp. 427-441, 2003.

[16] S. Leven, J.-C. Zufferey, and D. Floreano, "A minimalist control strategy for small UAVs," in Proceedings of the IEEE/RSJ International Conference on Intelligent Robots and Systems, 2009, pp. 2873-2878.

[17] S. Hauert, J. Zufferey, and D. Floreano, "Evolved swarming without positioning information: an application in aerial communication relay," Autonomous Robots, vol. 26, no. 1, pp. 21-32, 2009.

[18] S. Hauert, L. Winkler, J. Zufferey, and D. Floreano, "Ant-based swarming with positionless micro air vehicles for communication relay," Swarm Intelligence: Special Issue on Swarm Robotics, vol. 2, no. $2-4$, pp. $167-188,2008$. 